\title{
Nuclear cardiology in India and the developing world: opportunities... and challenges!
}

\author{
Vikram R. Lele, MD, DRM, DNB, ${ }^{\mathrm{a}}$ and Prem Soman, MD, PhD, FRCP ${ }^{\mathrm{b}}$
}

In the United States and other industrialized nations, myocardial perfusion SPECT (MPS) is widely utilized to diagnose coronary artery disease (CAD) and estimate risk of adverse cardiovascular outcomes. ${ }^{1}$ Using its established prognostic power, informed management decisions are made based on the assessed risk of cardiac death and nonfatal myocardial infarction. With this approach physicians are able to reserve invasive management strategies for patients at higher risk and thereby optimize the risk-benefit ratio for the patient. Importantly, a strategy that uses MPS to determine who needs coronary angiography has proven safe and costeffective. ${ }^{2}$ Based on three decades of clinical experience, an extraordinary volume of supporting data, and favorable reimbursement, approximately 7 million MPS are performed annually in the United States alone.

One would expect a diagnostic modality with these performance characteristics to be widely used in parts of the world where $\mathrm{CAD}$ is prevalent, but access to coronary angiography is limited. A case in point is India, where unique patterns of health care demand and supply exist, and which is representative of many "developing" countries currently experiencing remarkable rates of economic growth. While many hospitals in large urban cities in these countries offer advanced diagnostic and treatment facilities that compare favorably with the best in the world, there is often a striking dichotomy between health care facilities in urban and rural areas. While health care services in the major metropolitan cities are provided mostly on a fee for service basis (with a progressively increasing proportion subscribing to westernstyle heath insurance policies), the basic and meager health care available in the rural areas is provided by the government free of cost. However, even in the major

From the Division of Nuclear Medicine and PET/CT, ${ }^{\mathrm{a}}$ Jaslok Hospital and Research Center, Mumbai and Division of Cardiology, ${ }^{\mathrm{b}}$ University of Pittsburgh Medical Center, Cardiovascular Institute, Pittsburgh, PA.

Reprint requests: Prem Soman, MD, PhD, FRCP, Division of Cardiology, University of Pittsburgh Medical Center, Cardiovascular Institute, A-429 Scaife Hall, 200 Lothrop Street, Pittsburgh, PA 15213; somanp@upmc.edu.

J Nucl Cardiol 2009;16:348-50

$1071-3581 / \$ 34.00$

Copyright $(2009$ by the American Society of Nuclear Cardiology. doi:10.1007/s12350-009-9073-1 metropolitan areas where new advances in cardiac diagnostic imaging such as cardiac magnetic resonance (CMR) and computed tomographic coronary angiography (CTA) are quickly incorporated into clinical practice, radionuclide cardiac imaging techniques have been extremely slow to find widespread acceptance. This article will explore the current state of utilization of radionuclide cardiac imaging in developing countries, using India as the example, and will focus on (1) whether CAD demographics and health economics of these regions actually indicate a role of MPS, (2) factors influencing current trends of MPS utilization in these regions, (3) opportunities for optimizing MPS utilization, and (4) challenges of implementing MPS use in developing countries.

The developing world has a huge and increasing burden of CAD which is now the largest killer of its adult population. ${ }^{3,4}$ The most recent estimates indicate a CAD prevalence of $11 \%$ among adults in India, which is a 10 -fold increase over the past 40 years. ${ }^{5} \mathrm{~A}$ report by the World Health Organization indicates that by the end of this century, India will account for more than half of the CAD patients in the world. ${ }^{6}$ This staggering statistic is driven by an exponential increase in the number of patients with diabetes mellitus, with India poised to harbor $10 \%$ of the world's diabetics in the next decade or so, and a high prevalence of other traditional CAD risk factors. ${ }^{5,7}$ Patients from the Indian subcontinent with established CAD are younger than their western counterparts. ${ }^{8}$ CAD deaths in individuals aged 35-64 in India resulted in 9.2 million years of productive life lost in 2000 , which is $570 \%$ more than the corresponding figure for the United States. ${ }^{5}$ Thus, there is an obvious need for a robust infrastructure system to enhance preventive efforts, and to detect and treat CAD. In a system fraught with inequalities of the type mentioned above, noninvasive testing to optimize patient selection for more expensive and less available invasive diagnostic and treatment strategies could play a very important and effective role. This is essentially the most effective way to utilize MPS. Furthermore, in many eastern cultures there is an inherent reluctance to undergo invasive testing, with patients more likely to choose a noninvasive diagnostic strategy if one is available. Thus, the substrate for widespread use of MPS definitely exists in the developing world. 
A closer look at the current state of affairs, however, indicates significant underutilization of radionuclide cardiac imaging tests. There are an estimated 250 centers capable of performing MPS in India (personal communication from Nuclear Cardiology Society of India survey). The tracers used for MPS, including thallium-201, and the Tc-99m ligands sestamibi and tetrofosmin, are readily available in urban areas. However, in 2007, approximately 50,000 myocardial perfusion scans were performed (personal communication from an industry survey). With a population in excess of 1 billion, and an extraordinarily high prevalence of CAD $(11 \%)$, this is a strikingly low number compared to the 8 million annual MPS performed in the United States with a population of 303 million, and a $4.85 \%$ prevalence of CAD. ${ }^{9}$ The rapid percolation of advanced diagnostic and therapeutic modalities such as CTA, CMR, and state-of-the-art percutaneous coronary intervention into mainstream cardiology in the large cities indicates an abundance of highly skilled professionals and the availability of investment capital. For example, according to the national registry of the Cardiological Society of India, the number of coronary interventions has doubled from 2002 to $2005 .{ }^{10}$ Advanced electrophysiological procedures including ablation therapy are offered in more than 40 centers in the country. ${ }^{10}$ A recent publication referred to the availability of facilities for robot-assisted, endoscopic coronary artery bypass surgery in two hospitals in the capital city of New Delhi. ${ }^{10}$ Therefore, the strikingly low utilization of radionuclide-based cardiac imaging tests in the urban areas cannot be explained solely by financial considerations or the lack of availability of equipment or trained personnel.

In this regard, one important difference between India and the United States is that in India, radionuclide cardiac imaging is performed only by physicians trained in Nuclear Medicine as their primary specialty. Nuclear Medicine exists as an independent specialty, unaffiliated to Radiology. Certification in Nuclear Medicine is obtained after a two-year training period (Diploma in Radiation Medicine, DRM) or three-year training period (MD or a Diplomate of the National Board of Examinations, DNBE) followed by a licensing examination. Successful candidates are then authorized by the Atomic Energy Regulatory Board (AERB) to practice Nuclear Medicine at certified institutions and centers. Nuclear Cardiology training is offered as part of the training in general nuclear medicine, with the minimum required exposure to cardiac physiology and clinical cardiology. Training programs in Cardiology (equivalent to the fellowship programs in the United States) offer minimal or no exposure to Nuclear Cardiology procedures, and, indeed, there is no short pathway for a cardiologist to obtain a license from the AERB to perform radionuclide-based imaging tests. Given this situation, the practicing cardiologist has little incentive to pursue an interest in Nuclear Cardiology, and is often not fully familiar with the scope and potential of radionuclide cardiac imaging. This profoundly influences utilization of Nuclear Cardiology by cardiologists, the primary players in the field, who are more likely to choose familiar tests such as stress echocardiography and coronary angiography. This is perhaps the most important factor leading to the underutilization of radionuclide cardiac imaging in the urban areas. To promote nuclear cardiology in these areas, attention should be directed to the core curriculum of cardiology training programs, where dedicated nuclear cardiology training should be mandated to the same level as echocardiography and cardiac catheterization, as is the practice in the United States and England. For example, in the authors' institution at the University of Pittsburgh, all Cardiology fellows undergo 6 months of rotations through Nuclear Cardiology laboratory, leading to level II certification by the COCATS criteria. ${ }^{11}$ Such a mandate will, of course, require that institutions training Cardiology fellows be equipped with the facilities and personnel to provide such training. It will also require advocacy by leaders who influence health policy decisions.

In the smaller cities and rural areas, economic considerations play a much bigger role in deciding the level of health care facilities available. Hospitals in these areas generally lack sophisticated equipment and highly trained personnel. At the current time, patients from small cities and rural areas are likely to have more access to stress echocardiography, and sometimes even coronary angiography, than MPS. For patients paying out of pocket, these tests are also likely to be cheaper than MPS. Although a minority of government-run hospitals may provide MPS at a subsidized cost, the average cost to patients of MPS with a Tc-99m-based agent is approximately $\$ 180$ (compared to the average monthly middle-class income of about $\$ 500$ ). In addition, the uninterrupted availability of tracer in areas remote from big cities is also likely to be a problem. Thus, promoting Nuclear Cardiology in smaller cities and rural areas is likely to be more challenging, and will require investment in equipment and training of personnel, and the continuous supply of radionuclide tracers at an affordable cost.

In recent years there has been an attempt to promote nuclear cardiology and increase awareness of radionuclide-based cardiac imaging. The Nuclear Cardiology Society of India (NCSI) was formed in 1996, and is dedicated to this goal. It has already initiated a dialogue with the burgeoning health insurance industry to promote the use of nuclear cardiology for ischemia-guided coronary revascularization. In addition, India has two 
other national societies dedicated to nuclear medicine: The Society of Nuclear Medicine, India (SNMI, www.snmindia.org), and The Association of Nuclear Medicine Physicians of India (ANMPI, www.anmpi.org). The Asia-Pacific Society of Nuclear Cardiology (APSNC, www.apsnc.org) was formed in 2000 and is comprised of Nuclear Cardiologists and Nuclear Medicine physicians from Australia, Bangladesh, China, India, Indonesia, Japan, Korea, Malaysia, Pakistan, the Philippines, Singapore, and Thailand. The aim of the society is to promote the development of nuclear cardiology throughout the Asia-Pacific region as an appropriate and useful diagnostic tool in cardiovascular disease, increasing awareness and understanding of the role that nuclear cardiology can play in the management of heart disease, improving the quality of nuclear cardiology practice, supporting the training and teaching of nuclear cardiology, and fostering co-operation and fellowship between the nuclear cardiology communities in the region. Such concerted efforts on the part of national and international organizations to promote nuclear cardiology will undoubtedly go a long way in promoting its use in the developing world.

Recognizing the looming crisis posed by a staggering increase in $\mathrm{CAD}$ prevalence, the Indian government has initiated significant efforts and financial investment in the form of the National Program for Prevention and Control of Diabetes, Cardiovascular Diseases, and Stroke to tackle this threat. ${ }^{5}$ Thus, this is an opportune time to direct attention to the potential scope of nuclear cardiology. The American Society of Nuclear Cardiology could have an important role in this process.

\section{References}

1. Klocke FJ, Baird MG, Lorell BH, Bateman TM, Messer JV, Berman DS, et al. ACC/AHA/ASNC guidelines for the clinical use of cardiac radionuclide imaging-executive summary: A report of the American College of Cardiology/American Heart Association Task Force on Practice Guidelines (ACC/AHA/ASNC Committee to Revise the 1995 Guidelines for the Clinical Use of Cardiac Radionuclide Imaging). Circulation 2003;108:1404-18.

2. Shaw LJ, Hachamovitch R, Berman DS, Marwick TH, Lauer MS, Heller GV, et al. The economic consequences of available diagnostic and prognostic strategies for the evaluation of stable angina patients: An observational assessment of the value of precatheterization ischemia. Economics of Noninvasive Diagnosis (END) Multicenter Study Group. J Am Coll Cardiol 1999;33:661-9.

3. Leeder S, Raymond S, Greenberg H, Liu H, Esson K. A race against time. The challenge of cardiovascular disease in developing countries. New York, NY: Columbia University; 2005.

4. Reddy K, Shah B, Varghese C, Ramadoss A. Responding to the threat of chronic diseases in India. Lancet 2005;366:1744-9.

5. Reddy KS. India wakes up to the threat of cardiovascular diseases. J Am Coll Cardiol 2007;50:1370-2.

6. World Health Organization. The World Health Report 2005. Preventing chronic diseases: A vital investment. Geneva: WHO; 2005.

7. Yusuf S, Hawken S, Ounpuu S, Dans T, Avezum A, Lanas F, et al. Effect of potentially modifiable risk factors associated with myocardial infarction in 52 countries (the INTERHEART study): case-control study. Lancet 2004;364:937-52.

8. Joshi P, Islam S, Pais P, Reddy S, Dorairaj P, Kazmi K, et al. Risk factors for early myocardial infarction in South Asians compared with individuals in other countries. JAMA 2007;297:286-94.

9. American Heart Association. 2005 Heart and stroke statistical update. Dallas, TX: American Heart Association; 2004.

10. Chaturvedi V, Talwar S, Airan B, Bhargava B. Interventional cardiology and cardiac surgery in India. Heart 2008;94:268-74.

11. Cerqueira MD, Berman DS, Di Carli MF, Schelbert HR, Wackers FJ, Williams KA. Task force 5: Training in nuclear cardiology: Endorsed by the American Society of Nuclear Cardiology. J Am Coll Cardiol 2008;51:368-74. 